\title{
Aspectos práticos na indicação de revisão de hemostasia no pós-operatório imediato de cirurgia cardíaca
}

\author{
Luís Alberto DALLAN*, Sérgio de Almeida OLIVEIRA*, Dalton A. F. CHAMONE*, José Carlos Rossini \\ IGLÉZIAS*, Geraldo VERGINELLI*, Adib D. JATENE*
}

RBCCV 44205-97

\begin{abstract}
DALLAN, L. A.; OliVEIRA, S. A.; ChAMONE, D. A. F.; IGLÉZIAS, J. C. R.; VERGINELLI, G.; JATENE, A. D. - Aspectos práticos na indicaçāo de revisão de hemostasia no pós-operatório imediato de cirurgia cardiaca. Rev. Bras. Cir. Cardiovasc., 4(3): 225-230, 1989.

RESUMO: Foram estudados restrospectivamente os coagulogramas de 65 pacientes submetidos a revisão de hemostasia no pós-operatório imediato de cirurgia cardiaca. Os coagulogramas foram considerados compativeis ou não com distúrbio da coagulação sangüinea, por hematologista que desconhecia o achado cirúrgico. Dentre 51 pacientes cujos valores do coagulograma foram considerados normais, $46(90,2 \%)$ apresentavam sangramento ativo localizado, passivel de correção cirúrgica. Os $5(9,8 \%)$ demais pacientes apresentavam sangramento difuso. Dentre os 14 pacientes restantes, cujo coagulograma encontrava-se alterado, $8(57,2 \%)$ apresentavam sangramento difuso e $6(42,8 \%)$, localizado. Os autores concluem que, na persistência de sangramento excessivo pós-operatório e coagulograma normal, a revisão de hemostasia deverá ser prontamente indicada. Entretanto, se houver alteração do coagulograma, dever-se-à empregar, inicialmente, o tratamento clínico, desde que o ritmo do sangramento o permita. Caso o sangramento persista, mesmo com melhora do coagulograma, deverá ser pesquisada a presença de coágulos retidos no mediastino. Se positiva, deve-se programar nova toracotomia, visando à remoçāo de coágulos do mediastino.
\end{abstract}

DESCRIPTORES: sangue, coagulação; sangramento em cirurgia cardíaca; reoperação, cirurgia cardíaca.

\section{INTRODUÇĀO}

O sangramento excessivo constitui motivo de preocupação no pós-operatório imediato de cirurgia cardíaca. A dificuldade em padronizar-se o que seria aceitável como perda sangüínea decorre das variaçōes no porte e na duração do ato cirúrgico, além de alternâncias individuais de peso e superficie corpórea. A presença de distúrbios pré-existentes no sistema de coagulação e a ação residual de drogas que interferem nesse sistema também contribuem para dificultar essa avaliação.

As recentes técnicas de autotransfusão (COSGROVE et alii ${ }^{6}$ e LOVE et alii ${ }^{14}$ ) e o uso de bolsas coletoras que permitem o reaproveitamento do sangue proveniente dos drenos torácicos têm contribuído para atenuar a necessidade de transfusōes de sangue homólogo e seus derivados, mas não trazem solução para a causa básica do sangramento.

A incerteza da etiologia da síndrome hemorrágica pode comprometer a boa evolução pós-operatória do paciente. Isto porque podemos submête-lo a toracotomia desnecessária, caso o sangramento seja difuso e decorrente apenas de distúrbios na coagulação sangüínea. Por outro lado, a insistência em tratar-se clinicamente um paciente cujo sagramento somente cessará através

Trabalho realizado no Instituto do Coração do Hospital das Clínicas da Faculdade de Medicina da Universidade de São Paulo. São Paulo, SP, Brasil.

Recebido para publicação em 20 de outubro de 1989

- Do Instituto do Coração do Hospital das Clínicas da Faculdade de Medicina da Universidade de São Paulo.

Endereço para separtas: Luis Alberto Dallan. Av. Dr. Enéas Carvalho de Aguiar, 44. Divisão Cirúrgica. 05403 São Paulo, SP, Brasil. 
DALLAN, L. A.; OLIVEIRA, S. A.; CHAMONE, D. A. F.; IGLÉZIAS, J. C. R.; VERGINELLI, G.; JATENE, A. D. - Aspectos práticos na indicação de revisão de hemostasia no pós-operatório imediato de cirurgia cardiaca. Rev. Bras. Cir. Cardiovasc., 4(3): 225-230, 1989.

da ligadura de uma estrutura sangrante poderá expô-lo a depleçāo volêmica acentuada e desnecessária.

O estudo da coagulação sangüínea, nesses casos, assume papel preponderante. A determinação dos tempos de protrombina (TP), trombina (TT), tromboplastina parcial ativada (TPA) e contagem do número de plaquetas já permitem um diagnóstico inicial da causa do sangramento. Essa análise poderá ser complementada pela dosagem dos fatores II, V, VII, X, fibrinogênio e tempo de sangramento orientando a melhor forma de tratamento, seja clínico ou cirúrgico.

Nosso objetivo foi correlacionar dados de coagulação sangüinea com o achado cirúrgico, em pacientes submetidos a revisāo de hemostasia devido a sangramento excessivo no pós-operatório imediato de cirurgia cardíaca.

\section{CASUÍSTICA E MÉTODOS}

Foram analisados retrospectivamente os coagulogramas de 65 pacientes submetidos a cirurgia cardiaca com circulação extracorpórea (CEC), que necessitaram nova intervenção cirúrgica para revisão de hemostasia devido a sangramento excessivo.

A idade dos pacientes variou de 10 meses a 73 anos (média 53,4 anos); 49 eram do sexo masculino e 16 , do feminino. Os pacientes pertenciam a grupos variados, com a seguinte distribuição: $22(33,8 \%)$ coronariopatas, 26 (40\%) valvopatas, $10(15,4 \%)$ congênitos,

TABELA 1

DISTRIBUIÇÃO DOS PACIENTES POR GRUPOS

\begin{tabular}{lr}
\hline - Coronariopatas & $22(33,8 \%)$ \\
- Valvopatas & $26(40,0 \%)$ \\
- Congênitos & $10(15,4 \%)$ \\
- Coronariopatas + valvopatas & $3(4,6 \%)$ \\
- Outros & $4(6,2 \%)$ \\
\hline
\end{tabular}

TABELA 2

VALORES MÉDIOS DE TP, TPA E TT OBSERVADOS ENTRE

\begin{tabular}{l|c|c}
\multicolumn{3}{c}{ OS 65 PACIENTES } \\
\hline & Normal & Resultados \\
\hline Tempo de protrombina & $12,45 \mathrm{seg}$. & $19,28 \mathrm{seg}$. \\
\hline Atividade & $70-100 \%$ & $54,88 \%$ \\
\hline $\begin{array}{l}\text { Tempo tromboplastina } \\
\text { parcial ativada }\end{array}$ & $40 \pm 10 \mathrm{seg}$. & $56,65 \mathrm{seg}$. \\
\hline Relaçăo dos tempos & $1-1,25$ & 1,22 \\
\hline Tempo de trombina & $10 \pm 3 \mathrm{seg}$. & $12,61 \mathrm{seg}$. \\
\hline
\end{tabular}

TP = tempo de protrombina; TPA = tempo de tromboplastina parcial ativada; $T T=$ tempo de trombina.
$3(4,6 \%)$ coronariopatas e valvopatas e $4(6,2 \%)$ do grupo geral (Tabela 1).

Como rotina, os coagulogramas expressavam os valores do tempo de protrombina, tempo de tromboplastina parcial ativada, tempo de trombina e número de plaquetas. Dependendo desses resultados, os exames foram complementados com dosagem dos fatores II, V, VII, X e fibrinogênio.

Como norma geral, os pacientes que apresentavam tempo de trombina alterado foram inicialmente tratados com doses adicionais de sulfato de protamina. A reposiçāo de fatores de coagulaçāo deu-se pela administração de plasma fresco, complexos protombínicos ou crioprecipitados. $\mathrm{Na}$ deficiência plaquetária, qualitativa ou quantitativa, promoveu-se a transfusão de plaquetas. Após essas medidas, os coagulogramas foram repetidos.

\section{RESULTADOS}

Os resultados laboratoriais foram analisados por hematologista que desconhecia os achados cirúrgicos, tendo classificado os coagulogramas como compativeis, ou não, com distúrbios de coagulação.

Os valores médios dos tempos de protrombina, tempo de tromboplastina parcial ativada, tempo de trombina, obtidos nos 65 pacientes, assim como os valores desses exames considerados normais estāo relacionados na Tabela 2 .

Quatorze $(21,5 \%)$ pacientes tiveram seus coagulogramas considerados compativeis com distúrbio de coagulação. Destes, $8(57,2 \%)$ apresentavam sangramento difuso como achado operatório durante revisão de hemostasia (Tabela 3). Nos demais $6(42,8 \%)$ pacientes havia pontos localizados que justificavam o sangramento, sendo três em artéria intercostal, dois em sutura de aorta e um em sutura de átrio esquerdo (Tabela 4).

Cinqüenta e um $(78,5 \%)$ pacientes não apresentavam coagulograma com alteraçōes significativas. Destes, apenas $5(9,8 \%)$ tinham sangramento difuso. Nos $46(90,2 \%)$ restantes, foram localizados pontos de sangramento que justificavam plenamente o quadro hemorrágico (Tabela 5). Não houve mortalidade hospitalar dentre todos os pacientes.

\section{COMENTÁRIOS}

Os niveis de sangramento pós-operatório em cirurgia cardíaca constituem motivo de preocupação, especialmente em pacientes operados com o auxílio da circulaçāo extracorpórea. 
DALLAN, L. A.; OLIVEIRA, S. A.; CHAMONE, D. A. F.; IGLÉZIAS, J. C. R.; VERGINELLI, G.; JATENE, A. D. - Aspectos práticos na indicação de revisão de hemostasia no pós-operatório imediato de cirurgia cardiaca. Rev. Bras. Cir. Cardiovasc., 4(3): 225-230, 1989.

TABELA 3

PACIENTES COM COAGULOGRAMA ALTERADO E SANGRAMENTO DIFUSO

\begin{tabular}{|c|c|c|c|c|c|}
\hline & Paciente & $T P$ & $T P A$ & $T T$ & $\begin{array}{c}\text { Achado } \\
\text { operatorio }\end{array}$ \\
\hline 01 & A.B.V. & $(34 \%)$ & $73,0^{\prime} \quad(1,49)$ & $18,0^{\prime}$ & Difuso \\
\hline 02 & L.J.L. & $(24,5 \%)$ & $76,0^{\prime} \quad(1,52)$ & $11,5^{\prime}$ & Difuso \\
\hline 03 & W.S. & $(56,0 \%)$ & $72,0^{\prime} \quad(1,53)$ & 18,87 & Difuso \\
\hline 04 & V.G.G. & $40,0^{\prime} \quad(<10 \%)$ & Incoagulável & Incoagulável & Difuso \\
\hline 05 & J.A.M. & $23,3^{\prime} \quad(24,5 \%)$ & $53,3^{\prime} \quad(1,12)$ & $14,0^{\prime}$ & Difuso \\
\hline 06 & W.C. & $(28,5 \%)$ & $43,4^{\prime} \quad(0,90)$ & $12,3^{\prime}$ & Difuso \\
\hline 07 & L.A.C. & $56,0^{\prime} \quad(<10 \%)$ & $129,5^{\prime} \quad(2,64)$ & $15,5^{\prime}$ & Difuso \\
\hline \multirow[t]{2}{*}{08} & J.M.A. & $26,7^{\prime} \quad(20,5 \%)$ & $80,0^{\prime} \quad(1,64)$ & $16,5^{\prime}$ & Difuso \\
\hline & Média & $26,16^{\prime} \quad(28,3 \%)$ & $75,4^{\prime} \quad(1,55)$ & 15,23 & $x^{2}=1$ \\
\hline
\end{tabular}

- fora de estatística

$\mathrm{TP}=$ tempo de protrombina; TPA $=$ tempo de tromboplastina parcial ativada; $\mathrm{TT}=$ tempo de trombina.

TABELA 4

PACIENTES COM COAGULOGRAMA ALTERADO E SANGRAMENTO LOCALIZADO

\begin{tabular}{|c|c|c|c|c|c|c|}
\hline & Paciente & $T P$ & $T$ & $A$ & $T T$ & $\begin{array}{c}\text { Achado } \\
\text { operatório }\end{array}$ \\
\hline 01 & M.S. & $21,6^{\prime} \quad(28,0 \%)$ & $60,9^{\prime}$ & $(1,21)$ & $15,3^{\prime}$ & $\begin{array}{c}\text { Artéria } \\
\text { Intercostal }\end{array}$ \\
\hline 02 & N.A. & $29,1^{\prime} \quad(25,0 \%)$ & $63,1^{\prime}$ & $(1,31)$ & $10,3^{\prime}$ & $\begin{array}{c}\text { Artéria } \\
\text { Intercostal }\end{array}$ \\
\hline 03 & D.M. & $24,5^{\prime} \quad(33,5 \%)$ & $82,4^{\prime}$ & $(1,75)$ & $10,5^{\prime}$ & Sutura AE \\
\hline 04 & J.M.B. & 22,8 ' $\quad(27,0 \%)$ & $52,9^{\prime}$ & $(1,26)$ & $10,5^{\prime}$ & Sutura Ao \\
\hline 05 & E.H. & $22,3^{\prime} \quad(39,5 \%)$ & $48,9^{\prime}$ & $(1,05)$ & $12,0^{\prime}$ & $\begin{array}{c}\text { Artéria } \\
\text { Intercostal }\end{array}$ \\
\hline \multirow[t]{2}{*}{06} & V.F.O. & $17,5^{\prime} \quad(51,0 \%)$ & $49,5^{\prime}$ & $(1,01)$ & $15,3^{\prime}$ & Sutura Ao \\
\hline & Média & $22,97^{\prime}(24,0 \%)$ & 59,67 & $(1,27)$ & $12,32^{\prime}$ & \\
\hline
\end{tabular}

$\mathrm{AE}=$ aurícula esquerda $; \mathrm{Ao}=$ aorta $; \mathrm{TP}=$ tempo de protrombina $; \mathrm{TPA}=$ tempo de tromboplastina parcial ativada; $\mathrm{TT}=$ tempo de trombina.

A necessidade de anticoagulação sangüínea e a correta neutralização da heparina motivaram inúmeros estudos e controvérsias ${ }^{2,9} 12,13,16$. O controle do tempo de coagulaçāo ativado (TCA), descrito por HATTERSLEY ${ }^{11}$, em 1966, e sistematizado por BILL et alii ${ }^{3}$, em 1975 , em muito contribuiu para adequar a dosagem de heparina e de sulfato de protamina à sensibilidade e metabolismo de cada paciente ${ }^{17,19,21}$. O método foi rapidamente adotado em todos os grandes centros de cirurgia cardiaca e inúmeros estudos se sucederam, demonstrando sua eficácia na diminuição do sangramento e conseqüente redução na necessidade de sangue e seus derivados ${ }^{1}, 7,8,20$. A investigação pré-operatória de possiveis alteraçōes no sistema de coagulação e seu tratamento adequado têm sido uma rotina. Dessa maneira, pode-se melhor detectar as alteraçōes sangüíneas decorrentes da circulação extracorpórea e conseqüente consumo dos fatores de coagulação ${ }^{4}, 15,18$. Variações nos valores do tempo de protrombina e sua atividade, TTPA e TT, associadas à enumeração das plaquetas, complementadas pela dosagem dos fatores II, V, VII e $X$ e fibrinogênio, possibilitam identificar com precisão eventuais fatores etiológicos dos distúrbios de coagulação nesses pacientes ${ }^{10}$.

As primeiras horas de pós-operatório na unidade de recuperação cardíaca são críticas em relação aos sangramentos excessivos. O emprego de bolsas coleto- 
DALLAN, L. A.; OLIVEIRA, S. A.; CHAMONE, D. A. F.; IGLÉZIAS, J. C. R.; VERGINELLI, G.; JATENE, A. D. - Aspectos práticos na indicação de revisāo de hemostasia no pós-operatório imediato de cirurgia cardiaca. Rev. Bras. Cir. Cardiovasc., 4(3): 225-230, 1989.

TABELA 5

PACIENTES COM COAGULOGRAMA NÃO COMPATIVEL COM DISTÚRBIO DE COAGULAÇÃO

\begin{tabular}{|c|c|c|c|c|c|}
\hline & Paciente & $T P$ & $T P A$ & $T T$ & $\begin{array}{c}\text { Achado } \\
\text { operatorio }\end{array}$ \\
\hline 01 & C.S.F. & $12,5^{\prime} \quad(100 \%)$ & $51,8^{\prime} \quad(1,09)$ & $11,8^{\prime}$ & Difuso \\
\hline 02 & H.L.Z. & $14,7^{\prime} \quad(72,0 \%)$ & $49,9^{\prime} \quad(1,06)$ & $10,5^{\prime}$ & Difuso \\
\hline 03 & J.B.S. & $13,8^{\prime} \quad(98,0 \%)$ & $44,4^{\prime} \quad(0,90)$ & $12,0^{\prime}$ & Difuso \\
\hline $\begin{array}{l}04 \\
05\end{array}$ & $\begin{array}{l}\text { S.L. } \\
\text { S.G.A. }\end{array}$ & $\begin{array}{ll}14,8^{\prime} & (68,0 \%) \\
14,5^{\prime} & (75,0 \%)\end{array}$ & $\begin{array}{ll}48,0^{\prime} & (0,98) \\
44,5^{\prime} & (0,89)\end{array}$ & $\begin{array}{l}12,3^{\prime} \\
12,8^{\prime}\end{array}$ & $\begin{array}{l}\text { Difuso } \\
\text { Difuso }\end{array}$ \\
\hline \multirow{3}{*}{05} & Média & $14,06^{\prime}(82,6 \%)$ & $47,72^{\prime}(0,98)$ & $11,88^{\prime}$ & \\
\hline & 46 Pacientes & $T P$ & $T P A$ & $T T$ & $\begin{array}{c}\text { Achado } \\
\text { operatório }\end{array}$ \\
\hline & Média & $13,94^{\prime}(84,6 \%)$ & $55,79^{\prime}(1,08)$ & 11,02 & Localizado \\
\hline
\end{tabular}

$\mathrm{TP}=$ tempo de promtrombina; $\mathrm{TPA}=$ tempo de tromboplastina parcial ativada; $\pi \mathrm{T}=$ tempo de trombina.

ras, que possibilitam o reaproveitamento desse sangue, tem atenuado o problema, permitindo que sejam tomadas, paralelamente, medidas diagnósticas e terapêuticas. Entretanto, persiste o dilema do momento exato em que se deva intervir cirurgicamente no paciente. A possibilidade de coexistirem alteraçōes no coagulograma e sangramento localizado tem que ser considerada. Além disso, devemos lembrar o morbidade dessa depleção sangüinea sobre o sistema cardiocirculatório e demais órgãos vitais e os riscos inerentes a transfusōes sangüíneas. A retenção de coágulos no mediastino e cavidades pleurais pode, também, acentuar o consumo de fatores de coagulação e interferir mecanicamente na dinâmica circulatória, restringindo a expansibilidade cardiaca. Por outro lado, a reintervenção cirúrgica precoce e desnecessária também não é inócua, podendo acarretar problemas adicionais, especialmente em pacientes críticos ou depletados.

Preocupados com todas essas possibilidades, procuramos estabelecer uma correlação cirúrgico-laboratorial desses sangramentos, buscando avaliar o grau de informação proporcionado pela análise do coagulograma no diagnóstico etiológico das hemorragias pós-operatórias. O desconhecimento do achado cirúrgico pelo hematologista possibilitou que o julgamento de cada caso fosse isento de qualquer influência. Dentre os 65 pacientes estudados, em $51(75,5 \%)$ os coagulogramas obtidos antes da revisão cirúgica não demonstraram alteraçōes significativas. Destes, $46(90,2 \%)$ apresentavam sangramentos localizados, que justificavam a intensidade da hemorragia. A simples sutura do local sangrante permitiu o controle definitivo do sangramento. Em apenas $5(9,8 \%)$ pacientes desse grupo o sangramento foi considerado difuso, não tendo localização específica (Tabela 5). Esses resultados nos permitem concluir que, na vigência de coagulograma normal, as hemorragias importantes pós-operatórias são, em sua grande maioria, decorrentes de sangramentos de linha de sutura ou ramos vasculares e a revisão de hemostasia deve ser indicada precocemente.

Por outro lado, dentre os 14 pacientes cujo coagulograma mostrava-se alterado e, mesmo assim, foram submetidos a revisão de hemostasia, o achado operatório foi de sangramento difuso em $8(57,2 \%)$ (Tabela 3 ) e localizado nos $6(47,8 \%)$ restantes (Tabela 4$)$.

Esses resultados nos alertam para a possibilidade de sangramentos ativos e localizados mesmo diante de alteraçōes do coagulograma. Temos, entretanto, procurado corrigir as deficiências da coagulação antes de reintervir cirurgicamente nesses pacientes, salvo nos casos eın que a intensidade da hemorragia se torna incontrolável. A alteração isolada do TT é interpretada como excesso de heparina circulante e procuramos neutralizá-la através de doses adicionais de sulfato de protamina. Diante da normalização do $\Pi$ e persistência do sangramento, indicamos revisão cirúrgica. A alteração de TP em geral indica depleção de fatores vitamina $\mathrm{K}$ dependentes, principalmente com o fator $V$ em niveis normais. Caso sua administração leve a melhora no TP e permaneça o sangramento, também indicamos a reoperaçāo. A experiência tem demonstrado que sangramentos localizados podem levar ao acúmulo de coágulos na cavidade pericárdica. Desenvolve-se, então, fibrinólise seletiva e, posteriormente, sistêmica, em razāo do excesso de plasmina, o que acarreta alteraçōes dos fatores de coagulação e conseqüente sangramento difuso. Temos, nesses casos, indicado nova toracotomia para retirada dos coágulos e, eventualmente, administrar localmente o ácido tranexâmico ou epsilon aminocapróico, 
DALLAN, L. A.; OLIVEIRA, S. A.; CHAMONE, D. A. F.; IGLÉZIAS, J. C. R.; VERGINELLI, G.; JATENE, A. D. - Aspectos práticos na indicação de revisão de hemostasia no pós-operatório imediato de cirurgia cardiaca. Rev. Bras. Cir. Cardiovasc., 4(3): 225-230, 1989.

o que faz cessar o processo hemorrágico. Os dados apresentados e a experiência crescente com o manuseio de pacientes em pós-operatório imediato nos permitem estabelecer a seguinte conduta:

- Testes de coagulação alterados e sangramento pós-operatório intenso sugerem distúrbio de coagulaçāo sangüínea, devendo ser instituído tratamento clínico inicial.

- Testes de coagulação normais e sangramento pós-operatório excessivo são indícios de sangramento ativo localizado, sendo indicada revisão cirúrgica da hemostasia.

RBCCV 44205-97

DALLAN, L. A.; OLIVEIRA, S. A.; CHAMONE, D. A. F.; IGLÉZIAS, J. C. R.; VERGINELLI, G.; JATENE, A. D. - Practical aspects in the recommendation for reoperation in immediate postoperative phase of cardiac surgery. Rev. Bras. Cir. Cardiovasc., 4(3): 225-230, 1989.

ABSTRACT: Blood coagulation of 65 patients that needed reoperation to control excessive bleeding after cardiopulmonary bypass surgery was evaluated by hematologist that didn't know the reoperation findings. Forty-six $(90.2 \%)$ of 51 patients with normal tests before the reintervention had localizated bleeding, and five $(9.8 \%)$ had difuse hemorrhage. From 14 patients with abnormal coagulation tests, eight $(57.2 \%)$ had difuse bleeding and six (42.8\%) had a localizated bleeding. The authors concluded that with normal coagulation tests and excessive bleeding after cardiopulmonary bypass surgery a new reoperation is indicated. Otherwise, patients with abnormal coagulation tests can receive initial clinical treatment.

DESCRIPTORS: blood coagulation; bleeding in cardiac surgery; reoperation, cardiac surgery.

\section{REFERÊNCIAS BIBLIOGRÁFICAS}

1 ALBRECHTESEN, O. K.; STENBJERG, S.: BERG, E. Clinical experience with activated clotting time (ACT) controlled heparinization in open-heart surgery. Thorac. Cardiovasc. Surg., 30: 109-112, 1982.

2 BICK, R. L. - Alterations of hemostasis associated with cardiopulmonary bypass: pathophysiology, prevention, diagnosis and management. Semin. Thromb. Hemostasis, 3: 59-82, 1976.

3 BULL, S. B.; KORPMAN, A. R.; HUSE, H. W.; BRIGGS, D. B. - Heparin therapy during extracorporeal circulation: I. Problems inherent in existing heparin protocols. J. Thorac. Cardiovasc. Surg., 69: 674-684, 1975.

4 BURNS, E. R.; BILLETT, H. H.; FRATER, R. W.; SISTO, D. A. - The preoperative bleeding time as a predictor of postoperative hemorrhage after cardiopulmonary bypass. J. Thorac. Cardiovasc. Surg., 92: 310-312, 1986.

5 CASTAÑEDA, A. R. - Must heparin be neutralized following open-heart operation? J. Thorac. Cardiovasc. Surg., 52: 716-724, 1966.

6 COSGROVE, D. M.; AMIOT, D. M.; MESERKO, J. J. An improved technique for autotransfusion of shed mdiastinal blood. Ann. Thorac. Surg., 40: 519-520, 1985.

7 ESPOSITO, R. A.; CULLIFORD, A. T.; COLVIN, S. B.; THOMAS, S. J.; LACKNER, H.; SPENCER, F. C. -
The role of the activated clotting time in heparin administration and neutralization for cardiopulmonary bypass. J. Thorac. Cardiovasc. Surg., 85: 174-185, 1983.

8 ÉVORA, P. R. B.; BRASIL, J. C. F.; OTAVIANO, A. G.; MARGARIDO, E. A.; RIBEIRO, P. J. F.; REIS, C. L.; SILVA, J. R. P.; SECCHES, A. L. - Controle de coagulação em circulação extracorpórea: importância do emprego rotineiro do tempo de coagulação ativado. Arq. Bras. Cardiol., 37: 89-93, 1981.

9 FRIESEN, H. R. \& CLEMENT, A. J. - Individual reponses to heparinization for extracorporeal circulation. J. Thorac. Cardiovasc. Surg., 72: 875-978, 1976.

10 HARKEN, L. A.; MALPASS, T. W.; BRANSON, H. E.; HESSEL, E. A.; SLICHTER, S. J. - Mechanism of abdominal bleeding in patients undergoing cardiopulmonary bypass: acquired transient platelet dysfunction associated with selective $\propto$-granule release. Blood, 56 : 824-834, 1980.

11 HATTERSLEY, P. C. - Activated coagulation time of whole blood. JAMA, 196: 436-440, 1966.

12 JABERI, M.; BELL, W. R.; BENSON, D. W. - Control of heparin therapy in open-heart surgery. J. Thorac. Cardiovasc. Surg., 67: 133-141, 1974.

13 JOBES, D. R.;SCHWARTZ, A. J.; ELLISON, N.; ANDREWS, R.; RUFFINI, R. A.; RUFFINI, J. J. - Monitoring heparin anticoagulation and its neutralization. Ann. Thorac. Surg., 31: 161-166, 1981. 
DALLAN, L. A.; OLIVEIRA, S. A.; CHAMONE, D. A. F.; IGLÉZIAS, J. C. R.; VERGINELLI, G.; JATENE, A. D. - Aspectos práticos na indicação de revisão de hemostasia no pós-operatório imediato de cirurgia cardiaca. Rev. Bras. Cir. Cardiovasc., 4(3): 225-230, 1989.

14 LOVE, T. R.; HENDREN, W. G.; O'KEEFE, D. D.; DAGGETT, W. M. - Transfusion of predonated autologous blood in elective cardiac surgery. Ann. Thorac. Surg., 43: 508-512, 1987.

15 PALMER, R. L. - Laboratory diagnosis of bleeding disorders: basic screening tests. Lab. Diag., 76: 137-148, 1984.

16 PAPACONSTANTINOU, C. \& RADEGRAN, K. - Use of activated coagulation time in cardiac surgery. Scand. J. Thorac. Cardiovasc. Surg., 15: 213-215, 1981.

17 PICCIONI, M. A.; PICCIONI, J. L.; BRANDĀO NETO, M.; BUBIEUX, W. K.; AULER Jr., J. O.; AMARAL, R. V. G.; CHAMONE, D.; JATENE, A. D. - Neutralização da heparina "in vivo", após circulaçăo extracorpórea: estudo comparativo de efeitos de duas preparaçōes de protamina e da validade de controle pelo tempo de coagulação ativada (TCA). Rev. Bras. Anest., 36 : 363-372, 1986.
18 RAMSEY, G.; ARVAN, D. A.; STEWART, S.; BLUMBERG, N. - Do preoperative laboratory tests predict blood transfusion needs in cardiac operations? J. Thorac. Cardiovasc. Surg., 85: 564-569, 1983.

19 SCOTT, J. A.; BERENSTEIN, A.; BLUMENTHAL, D. Use of activated coagulation time as a measure of anticoagulation during interventional procedures. Radiology, 158: 849-850, 1986.

20 UMLAS, J.; TAFF, R. H.; GANVIN, G.; SWIERK, P. Anticoagulant monitoring and neutralization during open-heart surgery: a rapid method for measuring heparin and calculating safe reduced protamine doses. Anesth. Analg., 62: 1095-1099, 1983.

21 ZAJTCHUK, R.; COLLINS, G. J.; SCHUMANN, C. F.; HOLLEY, P. W.; HAMAKER, W. R. - Coagulation abnormalities in patients undergoing myocardial revascularization. J. Thorac. Cardiovasc. Surg., 75: 168-170, 1978. 